CAMS-98/03

HUB-EP-98/44

QMW-PH-98-33

hep-th/9807187

\title{
BPS BLACK HOLES IN N=2 FIVE DIMENSIONAL ADS SUPERGRAVITY
}

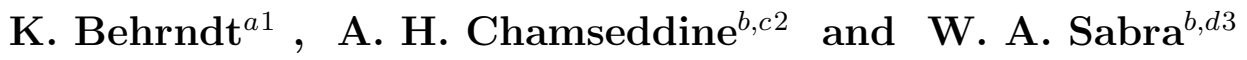 \\ ${ }^{a}$ Humboldt-Universität, Institut für Physik \\ Invalidenstraße 110, 10115 Berlin, Germany \\ ${ }^{b}$ Center for Advanced Mathematical Sciences, American University of Beirut, Lebanon. 1 \\ ${ }^{c}$ Institute for Theoretical Physics, ETH Zuerich Switzerland. \\ ${ }^{d}$ Physics Department, Queen Mary and Westfield College, \\ Mile End Road, E1 4NS, London, United Kingdom
}

\begin{abstract}
BPS black hole solutions of $U(1)$ gauged five-dimensional supergravity are obtained by solving the Killing spinor equations. These extremal static black holes live in an asymptotic $A d S_{5}$ space time. Unlike black holes in asymptotic flat space time none of them possess a regular horizon. We also calculate the influence, of a particular class of these solutions, on the Wilson loops calculation.
\end{abstract}

\footnotetext{
${ }^{1}$ behrndt@qft2.physik.hu-berlin.de

${ }^{2}$ chams@layla.aub.edu.lb

${ }^{3}$ w.sabra@qmw.ac.uk

4 Permanent address
} 
In the past years a considerable amount of work has been devoted to establish a duality between supergravity and super Yang Mills theories. For example the conformal field theory $(C F T)$ living on the boundary of the five-dimensional anti-de Sitter space $\left(A d S_{5}\right)$ is expected to be dual (in certain limits) to the four-dimensional super Yang Mills theory. Since this conjecture has been made [1] and further developed in [2], five-dimensional anti-de Sitter spaces have received a great deal of interest.

The aim of this letter is to describe BPS black holes living in an asymptotic $A d S_{5}$ vacuum (for the $A d S_{4}$ case, Reissner-Nordström solutions have been discussed in [3] and non-abelian monopoles in [4]). To keep these solutions as general as possible we formulate them in terms of $D=5, N=2$ supergravity with an arbitrary prepotential, i.e. the $N=4,8$ black holes appear as a special subclass for special choices of the prepotential of the $N=2$ theory. Because the asymptotic vacuum should be $A d S_{5}$ instead of flat Minkowski space, we gauge a $U(1)$ subgroup of the $S U(2)$ automorphism group, which results in a scalar potential that becomes constant at infinity.

In the first part we will describe these black holes as solutions of the Killing spinor equations of gauged $D=5, N=2$ supergravity [5] and in the second part we ask for the modification of the Wilson-loop calculations as done in [6], [7], [8].

First, we briefly describe the theory of $N=2$ supergravity coupled to an arbitrary number $n$ of abelian supermultiplets. $N=2$ supergravity theories in five-dimensions can be obtained, for example, by compactifying eleven-dimensional supergravity on a CalabiYau 3-folds [9]. The massless spectrum of the compactified theory contains $\left(h_{(1,1)}-1\right)$ vector multiplets with real scalar components. Including the graviphoton, the theory has $h_{(1,1)}$ vector bosons. The theory also contains $h_{(2,1)}+1$ hypermultiplets, where $h_{(1,1)}$ and $h_{(2,1)}$, are the Calabi-Yau Hodge numbers. In what follows and for our purposes the hypermultiplets are switched off. The anti-de Sitter supergravity can be obtained by gauging the $U(1)$ subgroup of the $S U(2)$ automorphism group of the $N=2$ supersymmetry algebra. This gauging, which breaks $S U(2)$ down to $U(1)$ can be achieved by introducing a linear combination of the abelian vector fields present in the ungauged theory, i.e. $A_{\mu}=V_{I} A_{\mu}^{I}$, with a coupling constant $g$. To restore supersymmetry, $g$-dependent and gauge-invariant terms have to be added. In a bosonic background, this amounts to the addition of a scalar potential, (for more details see [10, 11]).

The bosonic part of the effective gauged supersymmetric $N=2$ Lagrangian which describes the coupling of vector multiplets to supergravity is given by

$$
\begin{aligned}
e^{-1} \mathcal{L} & =-\frac{1}{2} R-\frac{1}{4} G_{I J} F_{\mu \nu}{ }^{I} F^{\mu \nu J}-\frac{1}{2} g_{i j} \partial_{\mu} \phi^{i} \partial^{\mu} \phi^{j}+\frac{e^{-1}}{48} \epsilon^{\mu \nu \rho \sigma \lambda} C_{I J K} F_{\mu \nu}^{I} F_{\rho \sigma}^{J} A_{\lambda}^{k} \\
& +g^{2} V_{I} V_{J}\left(6 X^{I} X^{J}-\frac{9}{2} g^{i j} \partial_{i} X^{I} \partial_{j} X^{J}\right)
\end{aligned}
$$

where $R$ is the scalar curvature, $F_{\mu \nu}=2 \partial_{[\mu} A_{\nu]}$ is the Maxwell field-strength tensor and $e=\sqrt{-g}$ is the determinant of the Fünfbein $e_{m}^{a}$. f

\footnotetext{
${ }^{5}$ The signature $(-++++)$ is used. Antisymmetrized indices are defined by: $[a b]=\frac{1}{2}(a b-b a)$.
} 
The physical quantities in (四) can all be expressed in terms of a homogeneous cubic polynomial $V$ which defines "very special geometry" [12].

$$
G_{I J}=-\left.\frac{1}{2} \frac{\partial}{\partial X^{I}} \frac{\partial}{\partial X^{J}}(\ln V)\right|_{V=1}, \quad g_{i j}=\left.G_{I J} \partial_{i} X^{I} \partial_{j} X^{J}\right|_{V=1}, \quad\left(\partial_{i} \equiv \frac{\partial}{\partial \phi^{i}}\right) .
$$

For Calabi-Yau compactification

$$
V=\frac{1}{6} C_{I J K} X^{I} X^{J} X^{K}=X^{I} X_{I}=1
$$

$V$ is the intersection form, $X^{I}$ and $X_{I}$ correspond to the size of the 2 and 4-cycles and $C_{I J K}$ are the intersection numbers of the Calabi-Yau threefold.

Since we are interested in finding BPS solutions in the gauged theory, we display the supersymmetry transformation of the Fermi fields in a bosonic background

$$
\begin{aligned}
\delta \psi_{\mu} & =\left(\mathcal{D}_{\mu}+\frac{i}{8} X_{I}\left(\Gamma_{\mu}{ }^{\nu \rho}-4 \delta_{\mu}{ }^{\nu} \Gamma^{\rho}\right) F_{\nu \rho}{ }^{I}+\frac{1}{2} g \Gamma_{\mu} X^{I} V_{I}-\frac{3}{2} i g V_{I} A_{\mu}^{I}\right) \epsilon \\
\delta \lambda_{i} & =\left(\frac{3}{8} \partial_{i} X_{I} \Gamma^{\mu \nu} F_{\mu \nu}^{I}-\frac{i}{2} g_{i j} \Gamma^{\mu} \partial_{\mu} \phi^{j}+\frac{3}{2} i g V_{I} \partial_{i} X^{I}\right) \epsilon
\end{aligned}
$$

where $\epsilon$ is the supersymmetry parameter and $\mathcal{D}_{\mu}$ is the covariant derivative

The spherically symmetric BPS electric solutions can be obtained by solving for the vanishing of the gravitino and gaugino supersymmetry variation for a particular choice for the supersymmetry parameter. We impose the projection operator condition on the spinor $\epsilon$

$$
\epsilon=\left(i a \Gamma_{0}+b \Gamma_{1}\right) \epsilon,
$$

where $a^{2}+b^{2}=1$ and this breaks $N=2$ supersymmetry to $N=1$.

We briefly describe the procedure of obtaining solutions preserving $N=1$ supersymmetry. First we start with an ansatz for the metric and gauge field

$$
\begin{gathered}
d s^{2}=-e^{2 V} d t^{2}+e^{2 W}\left(d r^{2}+f^{2} r^{2}\left(d \theta^{2}+\sin ^{2} \theta d \phi^{2}+\cos ^{2} \theta d \psi^{2}\right)\right) \\
A_{t}^{I}=e^{-2 U} X^{I}
\end{gathered}
$$

where the functions $U, V, W$ and $f$ are functions of $r$, and $(\theta, \phi, \psi)$ are the polar coordinates of the 3 -sphere. As solution of the gauge field equations we find

$$
e^{2 U} X_{I}=\frac{1}{3} H_{I}
$$

where $H_{I}$ is a harmonic functions which depends on the electric charge $q_{I}$. The supersymmetry variation of the gaugino and the time component of the gravitino imply the following relations

$$
e^{2 V}=e^{-4 U} f^{2}, \quad e^{2 W}=e^{2 U} \frac{1}{f^{2}}
$$

\footnotetext{
${ }^{6}$ More detailed analysis will be given in $[11$.
} 


$$
a=-\frac{1}{f}, \quad b=-\frac{1}{f} g r e^{3 U}, \quad f^{2}=1+g^{2} r^{2} e^{6 U}
$$

where we used some relations of special geometry analog to the derivation in [13].

The time and spatial components of the gravitino transformation imply differential constraints on the Killing spinor. These are

$$
\begin{gathered}
\left(\partial_{t}-i g\right) \epsilon=0 \\
\left(\partial_{r}-\frac{i}{2 f}\left(\frac{1}{r}+3 U^{\prime}\right) \Gamma_{0}-\frac{1}{2}\left(\frac{1}{r}+U^{\prime}\right)\right) \epsilon=0 \\
\left(\partial_{\theta}+\frac{i}{2} \Gamma_{012}\right) \epsilon=0 \\
\left(\partial_{\phi}+\frac{i}{2} \sin \theta \Gamma_{013}-\frac{1}{2} \cos \theta \Gamma_{23}\right) \epsilon=0, \\
\left(\partial_{\psi}+\frac{i}{2} \cos \theta \Gamma_{014}+\frac{1}{2} \sin \theta \Gamma_{24}\right) \epsilon=0 .
\end{gathered}
$$

Going to the rescaled coordinates

$$
X^{I}=\mathcal{V}^{-\frac{1}{3}} Y^{I}
$$

where $\mathcal{V}=e^{3 U}$, one obtains the following solution!

$$
\begin{aligned}
\epsilon & =e^{i g t} e^{-\frac{i}{2} \Gamma_{012} \theta} e^{\frac{1}{2} \Gamma_{23} \phi} e^{-\frac{i}{2} \Gamma_{014} \psi} \varphi(r) \\
\varphi(r) & =\frac{1}{2} \frac{\mathcal{V}^{-\frac{1}{2}}}{\sqrt{g r}}\left(\sqrt{f+1}-\sqrt{f-1} \Gamma_{1}\right) e^{\frac{1}{2} \int^{r} d \bar{r}\left(\frac{1}{\bar{r}}+\frac{1}{3} \frac{\mathcal{V}^{\prime}}{\mathcal{V}}\right)}\left(1-i \Gamma_{0}\right) \epsilon_{0}
\end{aligned}
$$

where $f=\sqrt{1+g^{2} r^{2} \mathcal{V}^{2}}$ and $\epsilon_{0}$ is an arbitrary constant spinor. Thus, inserting all terms in our ansatz one obtains

$$
\begin{aligned}
d s^{2} & =-\mathcal{V}^{-4 / 3}\left(1+g^{2} r^{2} \mathcal{V}^{2}\right) d t^{2}+\mathcal{V}^{2 / 3}\left[\frac{d r^{2}}{1+g^{2} r^{2} \mathcal{V}^{2}}+r^{2}\left(d \theta^{2}+\sin ^{2} \theta d \phi^{2}+\cos ^{2} \theta d \psi^{2}\right)\right] \\
F_{t m}^{I} & =-\partial_{m}\left(\mathcal{V}^{-1} Y^{I}\right) \\
\mathcal{V} & =\frac{1}{6} C_{I J K} Y^{I} Y^{J} Y^{K}, \quad \frac{1}{2} C_{I J K} Y^{J} Y^{K}=H_{I}=3 V_{I}+\frac{q_{I}}{r^{2}}
\end{aligned}
$$

Note that the constant parts in the harmonic functions are given by $V_{I}$, which fixes the $U(1)$ that has been gauged. The only deviation from the ungauged case [15] comes via the function $f^{2}=1+g^{2} r^{2} \mathcal{V}^{2}$. This term however changes completely the singularity structure of the black hole solution. To investigate this in more detail we may consider simple cases were $\mathcal{V}$ can be written as

$$
\mathcal{V}=H^{n}=\left(1+\frac{q}{r^{2}}\right)^{n} \quad, \quad n=0,1,2,3 .
$$

\footnotetext{
${ }^{7}$ The Killing spinors for a general $A d S_{p} \times S^{q}$ geometry are also discussed in [14].
} 
Obviously, the first case $(n=0)$ defines the $A d S_{5}$ vacuum with no black hole. The cases of $n=1,2$ correspond to black holes with a singular horizon and they appear naturally as BPS solutions of $N=4,8$ supergravity. In both cases the scalars are either zero or blow up near the horizon. The last case $(n=3)$ is an example of a BPS black hole of $N=2$ supergravity, which seems to have a regular horizon at $r \simeq 0$. However this coordinate system is misleading. Defining

$$
\rho^{2}=r^{2}+q
$$

one finds

$$
\begin{aligned}
& d s^{2}=-e^{2 V} d t^{2}+e^{-2 V} \Delta^{-1} d \rho^{2}+\Delta \rho^{2} d \Omega_{3} \\
& e^{2 V}=\tilde{H}^{\frac{2 n}{3}}+g^{2} \rho^{2} \Delta \quad, \quad \Delta=\tilde{H}^{\frac{3-n}{3}} \quad, \quad \tilde{H}=1-\frac{q}{\rho^{2}}
\end{aligned}
$$

In the ungauged case $(g=0)$ the horizon is at $\tilde{H}=0$ (or $\left.\rho^{2}=q\right)$, which is regular in the case $n=3$ or $\Delta=1$. But taking into account the gauging the horizon disappeared $\left(e^{ \pm 2 V}\right.$ is finite at $\rho^{2}=q$ for $n=3$ ) and the singularity at $\rho=0$ becomes naked. For the other cases $(n=1,2)$ the horizon becomes singular. For $n=1$ the singular horizon is infinitely far away, i.e. a light signal $\left(d s^{2}=0\right)$ would need infinite time to reach any finite distance (null singularity). But for $n=2$ the distance to the singular horizon is finite. This is different to the ungauged case $(g=0)$, where all singular cases have null horizons. Note also, the naked singularity at $\rho=0$ for $n=3$ (i.e. $\Delta=1$ ) is only a finite distance away! Certainly, this makes this solution rather suspisious and to overcome this situation one should consider the non-extremal case.

Let us nevertheless ask, what is the influence of this black hole on the Wilson loops as calculated in [6], [7], 8]. For this we calculate the Nambo-Goto action for open strings that are attached to the asymptotic boundary

$$
S=\frac{1}{2 \pi \alpha^{\prime}} \int d \tau d \sigma \sqrt{\left|\operatorname{det} g_{\alpha \beta}\right|} \quad, \quad g_{\alpha \beta}=\partial_{\alpha} X \partial_{\beta} X^{N} G_{M N}
$$

where $G_{M N}$ is the 5 d metric. For the worldsheet coordinates we choose the gauge

$$
\tau=t \quad, \quad \sigma=\theta
$$

where $\theta$ is the polar angle in $\Omega_{3}$ (see figure and the eq. (77)). Obviously, the string will stretch inside the AdS space and thus its position is given by a function $f(\theta, \rho)=0$, where $\rho$ is the radial coordinate. This defining equation can also be expressed as $\rho=\rho(\sigma=\theta)$. For the induced metric we find therefore

$$
\begin{aligned}
& g_{\tau \tau}=\partial_{\tau} X^{M} \partial_{\tau} X^{N} G_{M N}=G_{00}=-e^{2 V} \\
& g_{\sigma \sigma}=\partial_{\sigma} \rho \partial_{\sigma} \rho G_{\rho \rho}+G_{\theta \theta}=\left(\rho^{\prime}\right)^{2} e^{-2 V} \Delta^{-1}+\rho^{2} \Delta
\end{aligned}
$$

and thus,

$$
S=\frac{1}{2 \pi \alpha^{\prime}} \int d \sigma d \tau \sqrt{\left(\rho^{\prime}\right)^{2} \Delta^{-1}+\rho^{2} e^{2 V} \Delta}
$$




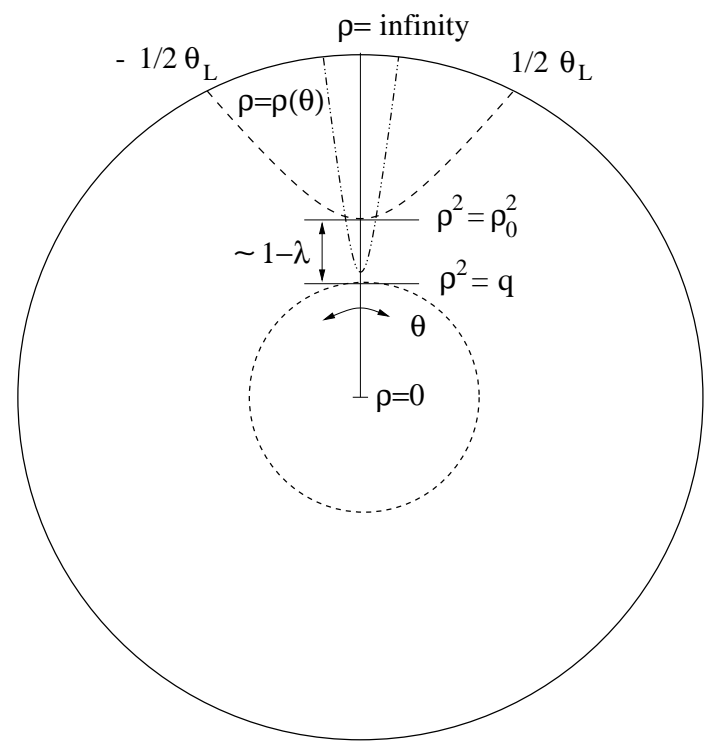

Figure 1: This is our geometry: a open string attached with the endpoints at asymptotic boundary, which is "perturbed" by a BPS black hole in the middle. The dotted line should indicate the horizon.

Following arguments given by Maldacena [0], we use the fact that the Lagrangian does not depend explicitly on $\theta$ and therefore

$$
c=\frac{\rho^{2} e^{2 V} \Delta}{\sqrt{\left(\rho^{\prime}\right)^{2} \Delta^{-1}+\rho^{2} e^{2 V} \Delta}} .
$$

The constant $c$ can be determined by going at the extremum $\rho_{0}$ where $\rho^{\prime}=0$ i.e.

$$
c^{2}=\left(\rho^{2} e^{2 V} \Delta\right)_{\rho=\rho_{0}} .
$$

In addition it follows from (15) that

$$
d \sigma=\frac{d \rho}{\rho \Delta e^{V} \sqrt{\frac{1}{c^{2}} \rho^{2} e^{2 V} \Delta-1}}=\frac{d y}{2 g \rho_{0} y^{\frac{n}{2}}(y-\lambda)^{\frac{3}{4} \delta} \sqrt{y^{\frac{2 n}{3}}\left(\frac{y-\lambda}{1-\lambda}\right)^{\delta}-1}}
$$

where $y=\left(\rho / \rho_{0}\right)^{2}, \lambda=q / \rho_{0}^{2}$ and $\delta=\frac{2(3-n)}{3}$ (see also the figure). Furthermore we consider here only the simplest case where $e^{2 V}=g^{2} \rho^{2} \Delta$ (i.e. we neglect the first term), which is a good approximation for the region $0 \ll q<\rho^{2}$. Integrating this equation yields the function $\rho=\rho(\sigma=\theta)$ that determines the position of the string in the AdS space. We can also calculate the distance between both endpoints on the boundary

$$
L=2 \int_{0}^{\frac{\theta_{L}}{2}} \sqrt{g_{\sigma \sigma} d \sigma^{2}}=\frac{(1-\lambda)^{\frac{\delta}{2}}}{g} \int_{1}^{\infty} \frac{d y}{y^{\frac{n}{3}}(y-\lambda)^{\frac{\delta}{2}} \sqrt{y^{\frac{2 n}{3}}(y-\lambda)^{\delta}-(1-\lambda)^{\delta}}} .
$$

\footnotetext{
${ }^{8}$ Note, we are dealing here with a different asymptotic geometry of $\mathbf{R} \times \mathbf{S}_{\mathbf{3}}$ (where $\mathbf{R}$ is the time).
} 
There are some interesting things to notice. First, for $n=3(\delta=0)$ all $\lambda$ dependence drops out and $L \sim 1 / g$ becomes independent of $\rho_{0}$ and the charge $q$, it scales only with cosmological constant. Thus it coincides with the case without black hole. Secondly, for $\delta \neq 0$ the integral is finite if $\lambda<1$, i.e. the string is away from the horizon. However, if the horizon comes close to string $(\lambda \rightarrow 1)$ the integral becomes divergent for $n=1$. However taking the pre-factor into account one finds that in this limit $L$ behaves like

$L \sim \frac{1}{g}(1-\lambda)^{1-\frac{\delta}{2}}$. Therefore, the string endpoints approach each other $L \rightarrow 0$ for $q \rightarrow \rho_{0}^{2}$ (see figure) if the horizon becomes large enough. This is different from the (neutral) Schwarzschild black hole, where $L \rightarrow \infty$ if the horizon comes close to the string $[\mathbb{8}$.

Finally, one may insert the solution (17) in the action and calculate the energy

$$
E=\frac{T}{2 \pi} \rho_{0}^{2} \int_{1}^{\infty} d y\left[\frac{y^{n / 6}(y-\lambda)^{\delta / 4}}{\sqrt{y^{2 n / 3}(y-\lambda)^{\delta}-(1-\lambda)^{\delta}}}-\frac{1}{\sqrt{y}}\right]
$$

where the last term is the subtraction of the infinite self-energy (see [7], [8]). Obviously, for $\lambda \rightarrow 1$ or $q \rightarrow \rho_{0}^{2}$ the energy remains finite although the string comes close to the singular horizon and it scales with the charge or the BPS mass of the black hole $E \sim q$. It is interesting to note that the energy is independent of $g$.

\section{Acknowledgements}

We thank P. Townsend for bringing to our attention the reference [16], which deals with black hole solutions in (anti) de Sitter background without vector multiplets.

\section{References}

[1] J. Maldacena, The large $N$ limit of superconformal field theory and supergravity, hep-th/9711200.

[2] S. S. Gubser, I. R. Klebanov and A. M. Polyakov, Gauge theory correlators from non-critical string theory, hep-th/9802109.

E. Witten, Anti-de Sitter space and holography, hep-th/9802150

[3] L. J. Romans, Nucl.Phys. B383 (1992) 395, hep-th/9203018.

[4] A. H. Chamseddine and M. S. Volkov, Phys.Rev. D57 (1998) 6242, hep-th/9711181; Phys.Rev.Lett. 79 (1997) 3343, hep-th/9707176.

[5] A. H. Chamseddine and H. Nicolai, Phys. Lett. B96 (1980) 89 and unpublished notes.

[6] S.-J. Rey, Macroscopic strings as heavy quarks in large $N$ gauge theory and anti-de Sitter supergravity, hep-th/9803001.

[7] J. Maldacena, Phys.Rev.Lett. 80 (1998) 4859, hep-th/9803002. 
[8] S.-J. Rey, S. Theissen and J.-T. Yee, hep-th/9803135

A. Brandhuber, N. Itzhaki, J. Sonnenschein and S. Yankielowicz, hep-th/9803138, hep-th/9803263.

[9] A. C. Cadavid, A. Ceresole, R. D'Auria, and S. Ferrara, Phys. Lett. B357 (1995) 76.

[10] M. Günaydin, G. Sierra, and P. K. Townsend, Nucl. Phys. B242 (1984) 244; Nucl. Phys. B253 (1985) 573.

[11] K. Behrndt, A. H. Chamseddine and W. A. Sabra, to appear

[12] B. de Wit and A. Van Proyen, Phys. Lett. 293 (1992) 94.

[13] A. H. Chamseddine and W. A. Sabra, Phys. Lett. B426 (1998) 36, hep-th/9811161.

[14] H. Lu, C.N. Pope and J. Rahmfeld, A Construction of Killing spinors on $S^{n}$, hep-th/9805151.

[15] W. A. Sabra, Mod. Phys. Lett. A13 (1998) 239, hep-th/9708103

[16] L.A.J. London, Arbitrary dimensional cosmological multi - black holes, Nucl. Phys. B434 (1995) 709. 